\title{
Privatización y globalización en los servicios públicos de acueducto y alcantarillado. Balance para el caso de Palmira, Valle del Cauca, Colombia*
}

* Este artículo es resultado de una investigación sobre "Asociaciones público-privadas para la prestación de servicios de agua y saneamiento" en varios países en desarrollo coordinada por "Tampere University of Technology" (Finlandia) y financiada por la "Foundation for Municipal Development" y la "Academy of Finland".

** M.Sc. Profesor - Instituto CIMARA - Facultad de Ingeniería - Universidad del Valle - Santiago de Cali, Colombia. e-mail: aleperez@univalle.edu.co.

Fecha de recepción: Septiembre 29 de 2004

Fecha de aprobación: Junio 10 de 2005

\section{RESUMEN}

Las políticas de privatización de los recursos hídricos y del sector de agua potable y saneamiento $(A \& S)$, son un instrumento eficaz que tiene el proceso de globalización para facilitar la canalización de los excedentes de liquidez de los países desarrollados hacia nuevas fuentes de acumulación de capital. En ese contexto, este artículo presenta un balance de la dinámica de privatización de los servicios de A\&S adelantado en Colombia, tomando como caso específico el proceso desarrollado en el municipio de Palmira (227 mil habitantes), Valle del Cauca. El artículo ayuda a ilustrar los diferentes efectos del proceso, mostrando que además de algunos beneficios 
asociados a la mejora en la eficiencia en la prestación del servicio, se generan a su vez importantes efectos perversos que es necesario considerar antes de continuar desarrollando procesos de este tipo en Colombia y América Latina.

Palabras clave: globalización, privatización, recursos hídricos, acueducto y alcantarillado, asociación público-privada, gobernabilidad.

\section{ABSTRACT}

The privatisation policies for the management of water resources, drinking-water and sanitation sectors are an efficient tool of globalisation to facilitate channelling the surplus from developed countries towards new sources of capital accumulation. In this context, this article presents an analysis of the results of the privatisation of drinking-water and sanitation services in Colombia, taking as a case study the process carried out in the city of Palmira $(227,000$ inhabitants) in the Valle del Cauca department. The article helps to illustrate the different effects of the process, showing that as the same time as some benefits have been produced to improvements in service -provision efficiency, other more damaging effects have also been generated, which should be taken into account before this process be fully developed in Colombia and Latin America.

Key words: globalization, privatization, water resources, water supply and sewerage, publicprivate partnership, governance.

\section{INTRODUCCIÓN}

En la actualidad, el agua dulce está siendo sometida a presiones crecientes que son producto del aumento de la población, el incremento de la actividad económica y el ascenso continuo de los niveles de consumo, aspecto que genera permanentes conflictos y una creciente competencia por el acceso y manejo del recurso hídrico, convirtiéndolo en un elemento estratégico donde su control es una fuente de poder, una clave para el desarrollo económico, un componente de tensión de la política social y sobre todo, un sector con importantes perspectivas de acumulación de capital para las empresas transnacionales. Precisamente, esta situación ha hecho que a nivel mundial, el sector haya desarrollado una importante transformación en los últimos diez años, mediante la cual se han creado los mecanismos legales e institucionales para facilitar un mayor acceso y control del sector privado al manejo y explotación del agua.

El presente ensayo trabajará sobre la base del estudio de caso de privatización de los servicios de $A \& S$ de la ciudad de Palmira, Valle del Cauca, contextualizándolo dentro del proceso de participación público-privada en la prestación de estos servicios en Colombia. Con relación al caso de Palmira, se hará un repaso histórico de la experiencia desde su inicio, hasta profundizar en el proceso de transformación empresarial de la segunda mitad de los noventa que dio origen a un esquema organizativo con amplia participación del sector privado a través de firmas francesas. Para brindarle al estudio un marco teórico de discusión, se presentarán dos enfoques alternativos que soportan tanto las bondades como los perjuicios de la privatización. Igualmente, se aborda como elemento contextual los diferentes esquemas de participación público-privada existentes.

\section{PLANTEAMIENTOS CONCEPTUALES DE LA PRIVATIZACIÓN}

Desde los años setenta y sobre todo ochenta, los gobiernos de América Latina y el Caribe han estado transfiriendo de diferentes formas muchas compañías públicas y otras instituciones estatales al sector privado. Tales transferencias han estado acentuadas en el sector manufacturero y otras actividades productivas, pero la privatización se ha extendido ahora a casi todos los sectores estratégicos de la economía, 
incluyendo la provisión de servicios básicos de agua. Este proceso es heredero de la mala negociación de la deuda externa de los años ochenta que facilitó la implementación de los denominados programas de "ajuste estructural" estimulados por los lineamientos de los préstamos condicionados del Banco Mundial (BM) y el Fondo Monetario Internacional (FMI). Por ello, frente al proceso privatizador, existe un consenso en tales organismos internacionales, en la mayor parte de los gobiernos de la región y en algunos teóricos, de que esto redundará en mejoras importantes en la eficiencia de la economía. Sin embargo, ese consenso se diluye cuando se interroga a buena parte de los usuarios de los servicios públicos y a otros teóricos que están preocupados por lo que pueda implicar el excesivo peso del sector privado en todas las esferas económicas en cuanto al acceso a tales servicios y la afectación de la democracia y el poder político. Explorar ambas vertientes de pensamiento puede ayudar a entender mejor las perspectivas de la privatización en Colombia.

\subsection{La privatización como un proceso necesario e inevitable:}

Desde el punto de vista de esta concepción, se señala que existe un consenso importante en cuanto a que la provisión de servicios productivos privados es la más efectiva herramienta para mejorar el bienestar económico. Las diferentes organizaciones internacionales (FMI, BM, OMC) y los académicos que respaldan esta opción, consideran que es necesario reemplazar el monopolio público por un monopolio privado regulado (MPR), esquema que mejorará tanto la eficiencia económica como el bienestar social a través de una mejor prestación de los servicios públicos. Esta doctrina tradicional, sustenta sus argumentos en varios puntos que se presentan en la Tabla 1.

Sin embargo, una condición necesaria para que opere adecuadamente el esquema de MPR, es el cambio en las funciones que el Estado debe asumir. Se requiere ahora un Estado menos interventor y más regulador; un Estado más diseñador de políticas y menos operativo en acciones; y bajo esta óptica son requeridas nuevas habilidades y conocimientos por parte de los funcionarios públicos (Lee y Jouravlev, 2000). Ello, junto al establecimiento de reglas de juego claras será suficiente para que la privatización mejore la gestión de los servicios de A\&S y alcance objetivos de universalización de coberturas.

Tabla 1. Elementos a favor de la privatización de los Servicios de A\&S

\begin{tabular}{|c|c|}
\hline Argumentos generales & Argumentos para países en desarrollo \\
\hline $\begin{array}{l}\text { Reducción de la interferencia política en } \\
\text { la toma de decisiones sobre la gestión } \\
\text { de los servicios de A\&S }\end{array}$ & $\begin{array}{l}\text { Aumento de la inversión extranjera y } \\
\text { mejoramiento de la balanza de pagos. }\end{array}$ \\
\hline $\begin{array}{l}\text { Derechos de propiedad en manos del } \\
\text { sector privado generan incentivos para } \\
\text { mejorar la eficiencia y la calidad de los } \\
\text { servicios prestados. } \\
\text { Mayor independencia del régimen } \\
\text { regulatorio que se perdía o capturaba en } \\
\text { el esquema de empresas públicas. } \\
\text { - Mayor efectividad en la administración } \\
\text { financiera que permite mejorar la gestión } \\
\text { de los servicios } \\
\text { Disminución de las tarifas por aumento } \\
\text { de la eficiencia. }\end{array}$ & $\begin{array}{l}\text { Reducción del déficit fiscal por la } \\
\text { venta de activos y disminución de la } \\
\text { presión sobre el presupuesto público } \\
\text { que requerían las empresas estatales. }\end{array}$ \\
\hline
\end{tabular}

Empero, esta línea conceptual no menciona la importante necesidad de fortalecer las organizaciones de usuarios con el fin de neutralizar los intereses privados y la incapacidad de vigilancia y control por parte del Estado.

\subsection{Visión crítica de la privatización}

La otra escuela de pensamiento, desde una concepción crítica, ve el proceso de privatización del sector de agua en general y de $A \& S$ en particular, como una oportunidad peligrosa que han creado los inversionistas internacionales para incrementar los niveles de acumulación de capital y de transferencia de recursos de los países en desarrollo a los países desarrollados. Los capitales transnacionales convierten el agua crecientemente escasa en un bien con alto valor económico y por lo tanto en un bien transable sujeto a apropiación, con lo que justifican el manejo racional del recurso que requiere tarifas y precios reales que faciliten el interés privado en su gestión, olvidándose de su carácter esencial 
Privatización y globalización en los servicios públicos de acueducto y alcantarillado. Balance para el caso de Palmira, Valle del Cauca, Colombia*

como bien social y ambiental. Esta estrategia del capitalismo pretende convertir en fuente de acumulación de capital los recursos naturales valorizándolos, para luego explotarlos, buscando ganancias en las externalidades negativas y positivas que se generan. Esto ha sido conocido como la estrategia de "enverdecimiento del capitalismo" que tiene como propósito abrir nuevos espacios de negocios (Bakker, 2002).

Dentro de este grupo de pensadores, algunos van más allá al afirmar que, "la privatización, se ha convertido en mucho más que una herramienta para beneficiar económicamente a unas pocas empresas multinacionales; también es utilizada para incrementar el peso político de las instituciones financieras internacionales y las multinacionales en todos los países del Sur con miras a promover un modo de vida que facilite el acceso a los mercados (Goldman, 2003). Así, se comprende la privatización como un componente más, pero fundamental, de la estrategia de globalización, la cual es entendida como el flujo de bienes, inversiones, producción y tecnología a través de los diferentes países, donde la acumulación de capital traspasa las fronteras con una tendencia importante a desplazar las estructuras previas asociadas con el EstadoMación (Petras y Veltmeyer, 2002). Luego, la privatización no representa un fenómeno aislado resultante de las circunstancias locales; ésta debe ser entendida como una parte de la estrategia global que responde a las órdenes de la banca internacional y es impulsada por consultores y entidades gubernamentales que conciben programas diseñados por estos grandes centros de poder de los países desarrollados. La privatización en este sentido es en esencia un acto político, que no se refiere a la creación de nuevos empleos, tasas más altas de ahorro y de inversión o nuevas fuerzas productivas. Así, aunque la privatización se establece como un medio para apoderarse de empresas, su objetivo final es irrumpir en los mercados como un recurso para eliminar las estructuras alternativas de producción que podrían competir con un mundo de dominación imperial o desafiarlo, de tal manera se torne irreversible la transición hacia el capitalismo neoliberal (Petras y Veltmeyer, 2002). Esa es la razón para que los políticos de la privatización estén más preocupados por el ritmo y el alcance del proceso que por los resultados financieros iniciales de las empresas privatizadas; ello hace que la privatización tenga un carácter ideológico, en donde uno de sus costos más importantes es la pérdida de gobernabilidad de las autoridades locales y nacionales. Los efectos negativos señalados por esta escuela se pueden apreciar en la Tabla 2.

Tabla 2. Efectos de la privatización desde una visión crítica

-La privatización ha polarizado la estructura de clases a través de tres elementos:
i) Los grandes beneficios recibidos por los empresarios compradores; ii) Los costos
en términos de despidos para los trabajadores y, iii) Las altas tarifas para los
consumidores.
- Debilitamiento de la democracia y pérdida de supervisión legislativa sobre sectores
estratégicos.
-Privación a la economía nacional de una fuente de acumulación de capital, sobre
todo cuando los nuevos inversionistas envían al exterior sus ganancias.
Pérdida del Estado de una palanca estratégica para el desplazamiento de las
ganancias a nuevos sectores de la economía.
- Desarticulación de la economía nacional al concentrarse en la producción e
importación desde enclaves.
Procesos privatizadores con marcadas características de corrupción donde el
precio de la venta suele ser un precio político.
Promesas de nuevas inversiones y de reducción de tarifas incumplidas. La mayoría
de las nuevas inversiones proceden de la banca local, de la deuda reconvertida o
de beneficios reinvertidos.
- Balance comercial y de pagos de las empresas privatizadas deficitario.
- Balance de generación de empleos netos negativa.
.Limitados procesos de transferencia de tecnología y desplazamiento del
conocimiento local.

Fuente: Petras y Veltmeyer (2002). Ajustes nuestros.

Estas dos visiones planteadas, sirven para ayudar a orientar el balance del proceso de privatización desarrollado en Colombia y específicamente en la ciudad de Palmira. El horizonte que debe prevalecer es un modelo en el que las relaciones entre lo público y lo privado sean compatibles con las necesidades de la mayoría nacional y local. Antes de presentar los resultados del estudio de caso objeto del trabajo, veamos los diferentes esquemas de participación privada desarrollados en el sector de $A \& S$.

\section{ESQUEMAS DE PARTICIPACIÓN PRIVADAEN EL SECTOR DE A\&S}

La privatización es casi siempre entendida en términos de la venta de la propiedad pública, una 
fabrica, una mina, una aerolínea, al inversor privado. Este concepto implica necesariamente el traspaso de la propiedad de los activos del sector público al sector privado. Sin embargo, una definición más amplia de la privatización, relaciona la transferencia de los derechos de las ganancias netas generadas por una empresa del sector público al sector privado, lo cual en sí mismo no requiere un cambio en la propiedad (Hemming and Mansoor, 1988, citado por Lee y Jouravlev 2000). Bajo esta definición, hay muchas formas de transferir actividades desde el Estado a los individuos y no todas ellas involucran la transferencia de activos físicos.

La Figura 1, muestra los diferentes escenarios de arreglos institucionales que pueden ocurrir entre el sector público y el sector privado donde el principal eje que mueve el espectro de alternativas es el nivel de participación del capital privado o público tanto en la propiedad de los activos como en su administración. La gráfica ofrece una ilustración de los seis principales modos de gestión: administración pública directa (dependencias municipales), administración pública corporativizada (empresas municipales), compañía pública limitada (regida por el derecho privado y conocida como modelo holandés), empresa privada delegada (modelo francés), empresas comunitarias de usuarios y empresa privada directa (modelo inglés). Aunque quizá este gráfico no es estrictamente correcto en términos de la teoría económica, la figura se aproxima bastante a la naturaleza real de los arreglos institucionales actuales (Braadbaart et al, 1999).

En ese contexto, el sector privado puede participar en un amplio rango de alternativas institucionales que se mueven en una línea continua que va entre la casi completa responsabilidad del sector público, donde el sector privado participa sólo en contratos de prestación de servicios, pasando a responsabilidades compartidas tales como las concesiones, arrendamientos, contratos de riesgo compartido, para finalizar en una opción de absoluta responsabilidad del sector privado. En el caso de la gestión del agua, el acto de privatización más significativo podría ser la concesión de los derechos de propiedad sobre el recurso hídrico, como en el caso de Chile ${ }^{2}$ (Lee y Jouravlev, 2000). Además, estas opciones pueden actuar en forma combinada y en cada una de las etapas del proceso de prestación del servicio tales como: captación y tratamiento; distribución; comercialización y cobro del servicio; transporte y disposición de aguas residuales y tratamiento de las mismas.

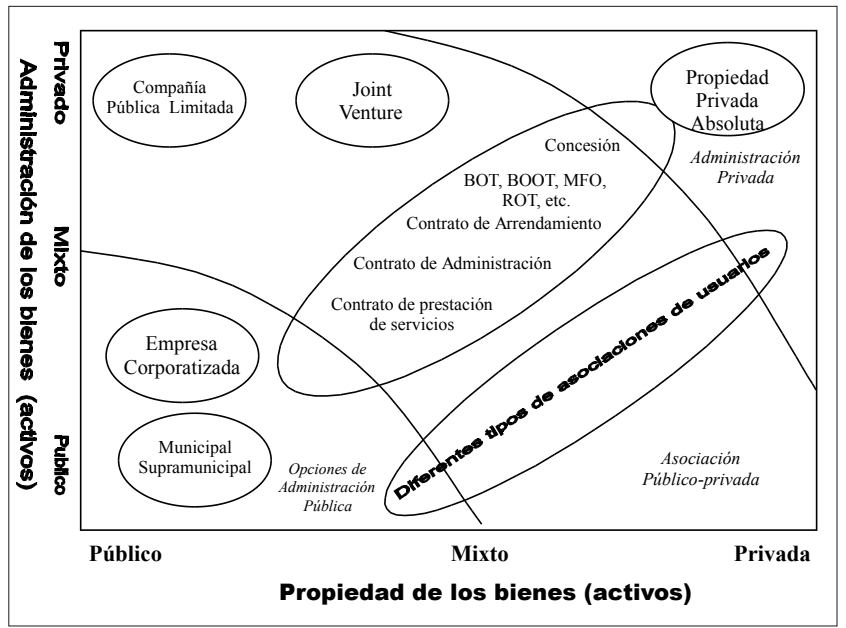

Figura 1. Modelos básicos de organización del sector de A\&S Fuente: Instituto UMESCO-IHE (1999).

Sin embargo, es importante resaltar que este esquema presentado no recoge las diferentes implicaciones económico-políticas asociadas al tipo de capital privado implicado en el proceso de privatización. Los resultados y efectos a nivel local y nacional son muy diferentes dependiendo de si el capital invertido es de carácter nacional o de

\footnotetext{
2 El "Código de Aguas de Chile" de 1981, consagra, con respaldo Constitucional, los derechos de los particulares sobre las aguas, otorgando a los titulares la propiedad sobre ellos. Esto permite a los propietarios de tales derechos una total y permanente libertad para el uso del agua a que se tiene derecho, pudiendo los titulares de derechos de agua, entre otras cosas: (i) usarlos o no, y destinarlos a las finalidades o tipos de uso que deseen; (ii) transferirlos, en forma separada de la tierra, para utilizarlos en cualquier otro sitio; y, (iii) comercializarlos a través de negociaciones típicas de mercado (vender, arrendar, hipotecar, etc.). Todas estas concesiones se otorgan con pocas y débiles normas regulatorias (Dourojeanni y Jouravlev, 1999).
} 
carácter foráneo. Precisamente, este caso analizado resalta las implicaciones del proceso privatizador cuando el capital involucrado es internacional, lo cual es casi la regla general para América Latina $(\mathrm{AL})$ dada las altas inversiones requeridas en este tipo de proyectos. De alguna manera, como lo señala Franceys (2000), la privatización de los servicios de A\&S en AL debería denominarse trasnacionalización.

\section{EVOLUCIÓN INSTITUCIONAL DE LOS SERVICIOS PÚBLICOS DE AGUA POTABLE Y SANEAMIENTO EN COLOMBIA}

Los antecedentes en la configuración de los sistemas de servicios públicos domiciliarios en A\&S en Colombia se remontan a las últimas décadas del siglo XIX, caracterizándose casi siempre por diferentes esquemas de asociación público-privada para su prestación. En ciudades grandes como Bogotá, Barranquilla y Medellín era corriente en un inicio, la intervención de las autoridades locales en la provisión colectiva de agua, mediante la conducción por canales rudimentarios o con revestimientos de lajas de piedra hacia el casco poblado, por lo general hacia pilas públicas (Jaramillo, 1995). Con el crecimiento de las ciudades, producto de la expansión de la economía cafetera en las primeras décadas del siglo $X X$, que se tradujo en la expansión del mercado interno, aparecen los primeros sistemas de acueducto que incluyen conexiones en los domicilios y en los lugares de trabajo. Los sistemas surgieron por iniciativa de empresarios privados en los centros urbanos de mayor dinamismo económico y demográfico, bajo la modalidad de contratos de concesión otorgados por el municipio: Barranquilla (1880), Bogotá (1886), Medellín (1891), Cartagena (1905), Pereira (1918) [Betancourt, 1998].

Acorde a Cuervo (1997) y señalado también por Fainboim y Rodríguez (2001), en el desarrollo posterior de estos sistemas se pueden distinguir al menos tres períodos. El primero que va de 1936 a 1950, está marcado por la creación del
Fondo de Fomento Municipal, evento que significó el cambio a un modelo de financiación con recursos de la Mación. El segundo período (19501986), se caracteriza por el fortalecimiento de la participación nacional que se extiende a la administración y operación de los sistemas a través del Insfopal (Instituto de Fomento Municipal) donde la política asistencialista del nivel central fue su característica. Y el último, que parte de la liquidación del Insfopal en 1986, está marcado por el proceso de descentralización y las amplias posibilidades de participación privada en la prestación directa de los servicios públicos.

El avance de esta última fase coincide con la puesta en práctica de un conjunto de reformas políticas y económicas importantes en América Latina conocidas como el "Consenso de Washington" a inicios de los noventa, caracterizadas por el impulso a reformas estructurales para disminuir el papel del Estado en la economía y la reducción del déficit fiscal con miras a mejorar los niveles de crecimiento económico. En este contexto, se expide la Ley 142 de 1994 de Servicios Públicos Domiciliarios, la cual genera una dinámica importante para la participación privada en la prestación de los servicios de A\&S. Este dinamismo, ha permitido alguna presencia significativa de inversión extranjera, aunque la misma resulta mucho más acentuada cuando se trata de los sectores eléctrico, gas y telefonía (ver Tabla 3). El bajo nivel de inversión extranjera en A\&S, cercano al 3\% de la inversión total en servicios públicos, está relacionado con el tipo de modalidad que ha caracterizado la participación privada en el sector: el Arrendamiento y la Concesión, en donde la inversión requerida no es significativa y se consigue básicamente a través del financiamiento de la banca local.

Sin embargo, es necesario resaltar que aunque la participación privada en la prestación de servicios de A\&S en Colombia parece ser pequeña al representar sólo un $4,4 \%$ del total de organizaciones prestadoras, ésta es más significativa cuando se mira la población servida, 
la cual es superior al 13\%. Esto es explicado porque el grueso de la inversión privada se concentra en las ciudades intermedias y grandes; es así que, el 83,7\% de la población beneficiada por la prestación privada de servicios de A\&S se encuentra en ciudades de más de 100 mil habitantes (ver Tabla 4). Igualmente, en este segmento de población servida es donde además se concentra la inversión foránea, de manera obvia pues es en gran escala la fuente principal de los grandes procesos de acumulación de capital; ejemplos de ello son los municipios de Barranquilla, Cartagena, Santa Marta, Montería; Palmira, Tuluá, Cartago y Girardot ${ }^{3}$.

Tabla 3.Inversión extranjera en Servicios Públicos y en Agua Potable y Saneamiento en Colombia (US\$ Millones)

\begin{tabular}{|c|c|c|c|}
\hline Año & Total & Electricidad, gas, agua & Agua Potable y Saneamiento \\
\hline 1990 & 230,3 & 0,0 & 0,0 \\
\hline 1992 & 350,5 & 53,0 & 1,5 \\
\hline 1994 & $1.389,5$ & 6,3 & 0,5 \\
\hline 1996 & $2.172,2$ & 145,4 & 3,6 \\
\hline 1998 & $3.952,2$ & 672,0 & 17,1 \\
\hline 2000 & 288,1 & 13,0 & 2,2 \\
\hline 2002 & 489,2 & 135,0 & 3,9 \\
\hline 2003 & 440,3 & 45,0 & 2,7 \\
\hline
\end{tabular}

Fuente: DMP (2003) y Banco de la República de Colombia (2003). Mota: Mo incluye inversiones en petróleo

Tabla 4. Municipios según rangos de población con participación privada en la prestación de servicios de A\&S en Colombia (2002) *

\begin{tabular}{|l|r|r|r|c|}
\hline \multirow{2}{*}{$\begin{array}{c}\text { Rangos de población de } \\
\text { las cabeceras municipales }\end{array}$} & \multicolumn{2}{|c|}{ Población beneficiada** } & \multicolumn{2}{c|}{ Múmero de empresas } \\
\cline { 2 - 5 } & \multicolumn{1}{c|}{$\#$} & \multicolumn{1}{c|}{$\%$} & $\#$ & $\%$ \\
\hline Hasta 5.000 hab & 40.684 & 0,74 & 14 & 22,95 \\
$5.001-12.500$ hab & 109.709 & 2,00 & 14 & 22,95 \\
$12.501-30.000$ hab & 145.195 & 2,65 & 7 & 11,48 \\
$30.001-100.000$ hab & 598.936 & 10,91 & 12 & 19,67 \\
$100.001-500.000$ hab & 2.662 .221 & 48,51 & 12 & 19,67 \\
> 500.001 hab & 1.931 .619 & 35,19 & 2 & 3,28 \\
$\begin{array}{l}\text { Población servida por prestación } \\
\text { privada }\end{array}$ & $\mathbf{5 . 4 8 8 . 3 6 4}$ & $\mathbf{1 0 0 , 0 0}$ & $\mathbf{6 1}$ & $\mathbf{1 0 0 , 0 0}$ \\
$\begin{array}{l}\text { Total población nacional y empresas } \\
\text { registradas }\end{array}$ & 42.000 .000 & & 1.390 & \\
$\begin{array}{l}\text { Participación población servida } \\
\text { por empresas privadas y frente } \\
\text { al total de empresas (\%) }\end{array}$ & & & & \\
\hline
\end{tabular}

* Incluye tanto las empresas con plena participación privada como las empresas mixtas.

** Población referente a 1997

Fuente: Mindesarrollo (2002). Cálculos propios.

\footnotetext{
3 La gran tradición de gestión pública en las ciudades más grandes del país (Bogotá, Medellín y Cali) y sus relativos buenos resultados en términos de la prestación del servicio, con un buen grado de excepción en Emcali, es lo que ha limitado la adquisición de tales empresas por capital extranjero. Sin embargo, en algunos casos, esta participación se hace en el manejo operativo de algunos componentes de los sistemas como las plantas de tratamiento de agua potable o aguas residuales; éste es el caso de Bogotá.
}

\section{LOS SERVICIOS PÚBLICOS DE AGUA Y ALCANTARILLADO EN LA CIUDAD DE PALMIRA}

\subsection{Contexto local}

Palmira es un municipio localizado en el sureste del Departamento del Valle del Cauca, siendo el segundo en importancia en la región, con una población de 267.665 habitantes en 1988, de los cuales 226.508 (83\%) viven en la cabecera municipal. Su principal actividad económica es la agro-industria, con énfasis en caña y producción de azúcar; también es importante la actividad metalmecánica. Debido a estas actividades industriales, los ingresos de la población son similares al promedio nacional. Su población es en general socio-económicamente homogénea: el $46 \%$ es de ingresos bajos (estrato 2); el $47.4 \%$ de ingresos medios ( 3 y 4 ); teniendo poca participación los ingresos muy bajos (estrato 1) y los ingresos altos (estrato 5 ) con $4.6 \%$ y $2 \%$ respectivamente.

\subsection{Evolución en la prestación de los servicios de a\&s en la ciudad de Palmira}

\section{Antecedentes (1850-1950)}

Con el fin de resolver los problemas de abastecimiento de agua de la localidad, a mediados del siglo XIX se construye por parte de la administración municipal un acueducto de calicanto que penetraba por la calle Real hasta la plaza principal donde se instaló una pila o surtidor. Más tarde se construyeron ramificaciones por varias calles y se instalaron surtidores en plazuelas y sitios públicos. Ya para 1925, cuando la localidad era más grande y requería de un acueducto metálico a presión, se constituye una sociedad anónima con aporte municipal de una cuarta parte del capital y el resto recursos del señor Alfonso Vallejo. Para 1926 se iniciaron los trabajos, pero en 1928 se liquidó la compañía sin haber terminado la obra, pasando todos los

\footnotetext{
4 El primer sistema de acueducto de la ciudad de Palmira, puede establecerse para el año de 1770, en cabeza de las autoridades provinciales de Popayán, época en que el país era colonia española.
} 
haberes y derechos al municipio. En ese mismo año, se constituyó una nueva sociedad entre el Municipio, el Departamento y veinte accionistas particulares que culminaron el acueducto en octubre de 1929. Una vez regularizado el servicio se construyeron algunos canales para alcantarillado que estaban generando problemas de salud pública y ya para 1938 se construyó la planta de potabilización por parte de la compañía (Raffo, 1956).

Puede observarse que a lo largo de este lapso de tiempo, la prestación de los servicios de A\&S en la ciudad de Palmira operó siempre bajo el esquema de una sociedad entre el sector público (municipio y departamento) y el sector privado.

\section{Las Empresas Municipales de Palmira, Empalmira (1950-1996)}

Luego de importantes esfuerzos del municipio y con el apoyo de la gobernación, se crearon en marzo de 1950 las Empresas Municipales de Palmira, teniendo como objetivo la prestación de los servicios de acueducto, alcantarillado, teléfonos, matadero municipal y plazas de mercado, siendo además esta infraestructura de propiedad del municipio. Durante este tiempo las Empresas Municipales se consolidan técnica, administrativa y políticamente siguiendo dinámicas similares a la de la mayoría de las empresas municipales de este tipo en el país. El principal cambio producido durante esta época, se originó en 1970 donde son transformadas en un Establecimiento Público del Orden Municipal con patrimonio independiente, aspecto que le permitió trasladar la propiedad de la infraestructura de manos del municipio a Empalmira.

Durante este tiempo, los servicios de acueducto, alcantarillado y teléfonos fueron prestados en forma eficiente teniendo balances financieros continuamente positivos, contrario a lo que sucedía con el Matadero y las Plazas de Mercado (Raffo Rivera, 1992). Igualmente los indicadores de cobertura de acueducto y alcantarillado alcanzaron para 1996, $86.7 \%$ y $83.3 \%$ respectivamente, niveles superiores al promedio nacional. En este período, Empalmira buscó la participación privada a través de contratos de consultoría para optimizaciones, planes maestros, ampliaciones y mejoras de los diferentes servicios prestados, teniendo prioridad las firmas locales y departamentales.

Sin embargo, al igual que el resto de empresas municipales del país, Empalmira no fue ajena al manejo politiquero, aspecto que se acentuó a partir de la segunda mitad de los años ochenta y principios de los noventa. Esta situación y los malos manejos administrativos, hicieron que la empresa comenzará a vivir dificultades que se manifestaron en déficit financieros continuos para acueducto y alcantarillado durante sus últimos cinco años de existencia (1992-1996) ${ }^{5}$. Esta situación se acompañó con un rezago significativo en las inversiones en acueducto $y$ alcantarillado y de un importante atraso tecnológico en telefonía. En situaciones más complejas se encontraba el matadero municipal y las plazas de mercado que siempre habían sido deficitarias. En enero de 1995 Empalmira presentaba un déficit acumulado de $\$ 9.000$ millones (US\$ 3,85 millones); cuentas por pagar por $\$ 13.000$ millones (US\$ 5,6 millones); 620 trabajadores en total de los cuales 301 estaban vinculados a acueducto y alcantarillado, y con un gran pasivo pensional (Empalmira, 1996).

Esta situación y el nuevo marco jurídico emitido (Ley 142/94 de servicios públicos domiciliarios) que obligaba a la transformación de las empresas municipales del país en Sociedades por Acciones con el propósito de promover una mayor

\footnotetext{
5 Para 1992, 1993, 1994, 1995 y 1996 el déficit anual para los servicios de acueducto y alcantarillado alcanzó las siguientes cifras respectivamente: $\$ 480$ millones (US\$ 205 mil); $\$ 408$ millones (US\$ $174 \mathrm{mil}) ; \$ 342$ millones (US\$ $146 \mathrm{mil}) ; \$ 812$ millones (US\$ 347 mil y $\$$ 168 millones (US\$ 72 mil), cifra que se redujo por el importante incremento tarifario de 1995 (EMPALMIRA, Informes anuales). Para estos años las actividades del servicio telefónico permitían subsidiar estos déficit y los de los otros dos servicios. El tipo de cambio con el que trabaja este documento es de $\$ 2340=$ US\$1, que es la tasa representativa de mercado promedio para mayo de 2005
} 
eficiencia en las mismas y un mejor comportamiento empresarial, impulsaron la creación de dos nuevas empresas de carácter mixto pero de manejo privado, sin la liquidación de Empalmira: Telepalmira para el manejo de la telefonía y Acuaviva para la operación de los sistemas de A\&S. Igualmente, fue liquidado el matadero y traspasadas las plazas de mercado al municipio. Esta transformación implicó la cancelación del contrato laboral de 620 trabajadores, de los cuales sólo 50 se pudieron reenganchar en las nuevas empresas ya conformadas, 20 en teléfonos y 30 en Acuaviva, con el correspondiente costo social para el resto de trabajadores y sus familias. Tal impacto se acentúa por la inexistencia de seguros de desempleo y una baja cobertura de la seguridad social en el país.

\section{La nueva empresa: Acuaviva S.A. (1997-hoy)}

\section{La negociación}

Acuaviva se constituye como una empresa operadora de carácter mixto en diciembre de 1996, por una cuantía autorizada de \$2.500 millones (US\$ 1,1 millones) y un capital suscrito de \$1.000 millones (US\$ 427 mil) y cuyos socios corresponden al municipio de Palmira (40\%); Lion Deus Aeuex (LYSA) -Francia (48\%) que a su vez es el socio operador del sistema; e, inversionistas privados de la región $(12 \%)^{6}$. En este esquema, Empalmira entrega la totalidad de las redes y la infraestructura física de acueducto y alcantarillado en condición de arrendamiento a Acuaviva por un período de quince años, teniendo el contrato un valor total cercano a los \$25.000 millones (US\$ 10.7 millones) pagaderos anualmente ${ }^{7}$; así mismo, a partir del año 11 (2007) y hasta finalizar el contrato, Empalmira recibirá el $60 \%$ de las utilidades. En ese momento, la totalidad de la

\footnotetext{
6 En la conformación inicial, la empresa francesa tenía el 36\% y los inversionistas privados colombianos el $24 \%$. Los inversionistas privados nacionales corresponden a empresas vallecaucanas vinculadas al sector azucarero y a firmas consultoras del sector de agua potable en el Valle del Cauca.

7 Se desconoce la existencia de un estudio técnico mediante el cual se haya calculado este valor y sus respectivas anualidades.
}

infraestructura, incluyendo las inversiones realizadas, será devuelta sin ningún costo a Empalmira.

Paralelamente al contrato de arrendamiento, se establecieron otros compromisos contractuales al interior de Acuaviva: Contrato de Manejo Operativo con LYSA, mediante el cual esta empresa se convierte en socia operadora, nombrando los principales directivos de Acuaviva y apoyándola con soporte técnico y administrativo. El valor de este contrato, que fue en dólares, alcanzó la suma de US\$ 4.610.000 pagaderos anualmente durante cinco años. Este contrato finalizó en 2002, siendo renovado a partir de junio de 2003 por diez (10) años más y por un valor adicional de 750 mil dólares anuales. Contrato de Asistencia Técnica con SAFEGE de Francia, también en dólares, por un valor de US\$ 6.409.000; este contrato por cinco años incluye: diseño de Planes Maestro para acueducto, alcantarillado y aguas lluvias; simulación de acuíferos y estudios hidrológicos; instalación de modelos de simulación de redes; implementación de sistemas de información geográfica y de gestión técnica centralizada y capacitación del personal en el exterior. Los compromisos de este contrato ya fueron finalizados a la fecha y no ha sido renovado. Contrato de Suministro de Equipo con AQUATECHMIQUE ${ }^{9}$, también francesa, por un valor mínimo de US\$ 2.060.000 ${ }^{10}$ y con una comisión de $20 \%$ del valor del contrato, es decir US\$ 412.000. Este contrato en la práctica nunca fue ejecutado. Así mismo, la puesta en marcha de este modelo requería de un costoso Plan de Inversiones para los quince años del contrato de arrendamiento, Plan que alcanzaba la suma de

8 En la práctica, la mitad del contrato se hizo en dólares y la otra mitad en pesos pero que se ajustan al tipo de cambio existente al final de cada año, con el fin de neutralizar el efecto devaluación. Así mismo, la mitad del valor del contrato es fijo y la otra mitad dependerá del cumplimiento de ciertos indicadores de gestión.

9 En esta investigación se desconoce la relación existente entre LYSA, SAFEGE y AQUATECHMIQUE. Estos costos son a precios FOB, es decir el valor en el puerto exportador, teniendo que asumir Acuaviva los costos y trámites de importación del equipo.

10 El valor final de este contrato, dependerá del valor definitivo de compra del equipo. 
US\$ 42.8 millones, divididos en US\$ 28.4 millones para ampliación y renovación de redes y equipos y US\$ 14.4 millones para pagar los contratos de asesoría con las firmas francesas. Estos recursos serían financiados a través de tarifas $^{11}$ y de un crédito con la banca nacional por US\$ 16.3 millones, el cual estuvo respaldado con la pignoración de los ingresos operativos de Acuaviva y la infraestructura de $A \& S$ de Empalmira.

Por su parte, con el propósito de administrar las acciones que poseía en las nuevas empresas operadoras (Telepalmira y Acuaviva), ejercer supervisión y control de tales servicios y asumir la operación de los mismos al vencimiento o terminación de los contratos, EMPALMIRA se transformó en el Instituto Financiero y de Fomento para el Desarrollo Municipal de Servicios Públicos, IMFIPAL, en enero de 1998. Sin embargo, el cumplimiento de esos objetivos se vio limitado por el enorme pasivo pensional que debió asumir la recién transformada empresa, aspecto que obligó a ordenar su liquidación en agosto de 1999, pasando todos los activos y pasivos a manos del Municipio (Infipal, 2001). Un esquema del modelo de participación privada en los servicios de A\&S en Palmira y de la evolución de las Instituciones Municipales encargadas de su vigilancia y control $(V \& C)$, se aprecia en la Figura 2.

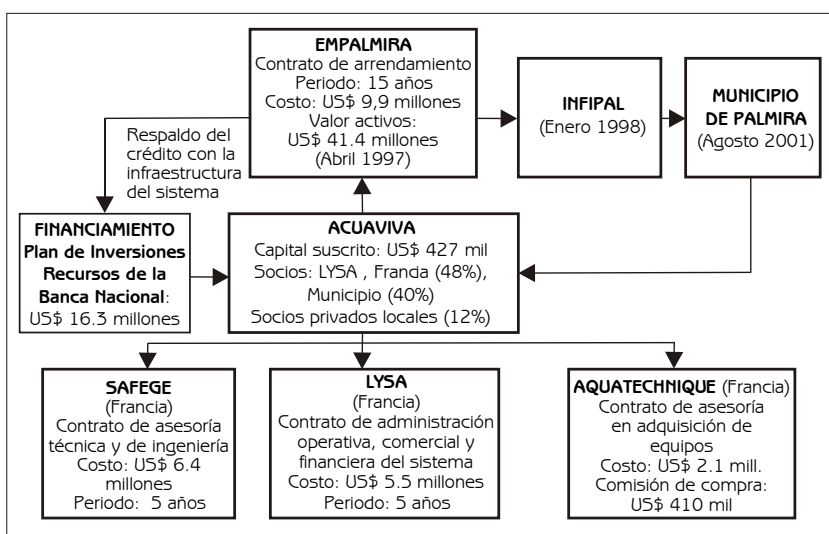

Figura 2.Esquema de Participación Privada en el servicio de A\&S en Palmira, Valle del Cauca, Colombia

Fuente: Autor.

11 Las tarifas habían sufrido un importante incremento por Empalmira en 1995 e igualmente se planteaban incrementos moderados para los 15 años del Plan y el desmonte de los subsidios extralegales acorde a la Ley.
En la práctica, este esquema de vinculación del sector privado ha generado un elevado control de la empresa por parte de uno de los socios, en este caso LYSA de Francia, siendo además un esquema que ha sido complejo y difícil de controlar por parte del socio propietario, Empalmira inicialmente y ahora el Municipio. Igualmente, se puede observar que es un contrato altamente ventajoso ("Ieonino" podríamos decir) para las firmas francesas y con muy poco rédito financiero para el socio estatal: mientras las primeras, con LYSA a la cabeza, invierten en el negocio US\$ 205 mil en la constitución de Acuaviva, obtienen con ello una retribución de US\$ 12.3 millones resultado de la suma de los tres contratos establecidos ${ }^{12}$, con lo cual, multiplica su inversión por 60 veces. Por su parte, el Municipio ${ }^{13}$, que aportó al negocio una suma equivalente a US\$ 41.571 .000 (que corresponden a US\$ 41.4 millones de los activos y US\$ 171 mil para la constitución de Acuaviva), obtendrá en los 15 años del contrato de arrendamiento una partida aproximada de US\$ 9,9 millones, cifra inferior a la obtenida por los franceses, sin incluir en ambos casos la repartición de utilidades. Es decir, el municipio multiplica su inversión por 0.24 veces. En términos anuales se observa un balance similar: mientras que los ingresos por año promedio de las firmas francesas ascienden a US\$ 2.46 millones, los ingresos anuales del municipio alcanzan sólo US\$ 660 mil durante los 15 años de operación del contrato. En este análisis no se incluye la renovación del contrato con LYSA por 10 años adicionales a partir del 2002.

Esta misma apreciación se puede extender al tema de la inversión extranjera, que es uno de los principales argumentos para defender la participación privada en servicios públicos en países en desarrollo (ver Tabla 1). Para este caso se puede decir que la misma fue mínima, dado además que el financiamiento del Plan de

\footnotetext{
12 Incluyendo la comisión de Aquatechnique y no la compra del equipo, que igualmente sería dinero que saldría del país, pues se establece que será importado.

13 Dada la transformación de Empalmira en Infipal y la liquidación de esta última pasando los activos al Municipio.
} 
Inversiones es obtenido con recursos internos de los usuarios (tarifas), de la banca nacional ${ }^{14} y$ aún del presupuesto municipal que se compromete a financiar las ampliaciones del sistema. Siendo así, la balanza de pagos del negocio, que equivale a lo que entra al país en inversión extranjera (US\$ 205 mil) menos lo que le sale por los contratos (US\$ 12.3 millones) $)^{15}$, es altamente deficitaria.

En conclusión, se puede afirmar que aunque se reconoce que es importante el "know how" y el respaldo de los socios franceses para consolidar la prestación de los servicios de acueducto y alcantarillado en la ciudad de Palmira para los próximos 15 años, ello no es óbice para mostrar que en este negocio salieron ampliamente beneficiados en perjuicio del municipio y de la comunidad palmirana que está representada en las autoridades municipales. Es importante tener presente, que en este proceso de participación del sector privado, el municipio actuó sin el respaldo necesario de las instituciones nacionales y que ello facilitó una negociación con claras debilidades éticas y administrativas.

\section{Situación actual de Acuaviva}

Acuaviva presta los servicios de acueducto y alcantarillado para la zona urbana del municipio, aunque también lo hace para dos pequeñas áreas rurales (Barranca y Guayabal), con un total de 164 empleados de los cuales 115 son de acueducto y 49 de alcantarillado, siendo además 107 operativos y 57 administrativos. Para junio de 2004, la empresa beneficiaba a 51.792 usuarios en acueducto y a 51.217 en alcantarillado, teniendo una cobertura de $100 \%$ para el primer servicio y de $98.8 \%$ para el segundo. Detalle sobre los significativos logros en la gestión operativa, administrativa y comercial de Acuaviva se aprecian en la Tabla 5.

\footnotetext{
14 Con relación al crédito obtenido, aparece adicionalmente una desventaja para el socio estatal, quien es el que ofrece la garantía física. Los socios privados no ofrecen ninguna dado que no la tienen.

15 En la práctica, no todo el valor de los contratos es repatriado al país de origen de los socios extranjeros, dado que en la ejecución de los mismos existen algunos pagos como salarios y otros rubros menores que se quedan en el país. Igualmente, habría que descontar los U\$ $410 \mathrm{mil}$ del contrato con AQUATECHNIQUE que no se ejecuto y sumar el nuevo contrato firmado con LYSA para continuar operando el sistema durante los próximos 10 años.
}

Tabla 5. Indicadores operativos y de gestión en Acuaviva (1998-2004)

\begin{tabular}{|c|c|c|c|c|c|c|c|}
\hline Conceptos & Dic-98 & Dic-99 & Dic-00 & Dic-01 & Dic-02 & Dic-03 & \begin{tabular}{|l|}
$J u n-04$ \\
\end{tabular} \\
\hline Agua no contabilizada acumulada & $31,6 \%$ & $35,9 \%$ & $35,1 \%$ & $32,5 \%$ & $25,4 \%$ & $26,2 \%$ & $24,8 \%$ \\
\hline Índice de recaudo acumulado & $84,2 \%$ & $91,8 \%$ & $97,2 \%$ & $98,1 \%$ & $103,6 \%$ & $98,8 \%$ & $98,8 \%$ \\
\hline Mo. suscriptores acueducto & 47,185 & 47,629 & 48,396 & 49.230 & 49.852 & 51.259 & 951.792 \\
\hline Mo. suscriptores alcantarillado & 46,374 & 46,991 & 47,924 & 48.739 & 49.315 & 50.692 & 51.217 \\
\hline \multicolumn{8}{|l|}{ Cobertura del servicio } \\
\hline Índice de cobertura acueducto & $98 \%$ & $97,1 \%$ & $96,9 \%$ & $96,8 \%$ & $99,7 \%$ & $100,0 \%$ & $100,0 \%$ \\
\hline Îndice de cobertura alcantarillado & $96 \%$ & $95,8 \%$ & $96,0 \%$ & $95,8 \%$ & $98,7 \%$ & $98,9 \%$ & $98,9 \%$ \\
\hline $\begin{array}{l}\text { Mo. De medidores instalados en } \\
\text { operación }\end{array}$ & MD & 39,392 & 46,667 & 47.793 & 49.586 & 51.039 & 51.582 \\
\hline Cobertura de medición eficiente & MD & $82,71 \%$ & $96,43 \%$ & $97,1 \%$ & $99,5 \%$ & $99,6 \%$ & $99,6 \%$ \\
\hline Mo. De quejas y reclamos recibidos & 9.766 & 13.710 & $\begin{array}{c}0,4510 \\
13.451\end{array}$ & 8.354 & $\begin{array}{l}99,519 \\
7.519\end{array}$ & $\frac{99,690}{4.690}$ & $\frac{99,010}{2.073}$ \\
\hline Mo. Quejas y Reclamos Aceptados & 4.034 & 6.739 & 2.578 & 966 & 978 & 837 & 250 \\
\hline $\begin{array}{l}\text { Plazo promedio instalación conexión } \\
\text { en días }\end{array}$ & MD & 7,85 & 6,62 & 6,45 & 6,06 & 3,78 & 2,79 \\
\hline $\begin{array}{l}\text { Longitud de red de alcantarillado } \\
\text { limpiada (m/año) }\end{array}$ & 172.331 & 45.393 & 121.573 & 166.744 & $\mid 192.046$ & 222.086 & 103.808 \\
\hline $\begin{array}{l}\text { \% acumulado red alcantarillado } \\
\text { limpiada año }\end{array}$ & $54 \%$ & $45 \%$ & $37,70 \%$ & $50,4 \%$ & $57.5 \%$ & $65,8 \%$ & $30,2 \%$ \\
\hline \multicolumn{8}{|l|}{ Rotación Cartera } \\
\hline Rotación Cartera global días & 58 & 103 & 96 & 84 & 64 & 60 & 58 \\
\hline Rotación cartera Particulares en días & 35 & 74 & 59 & 48 & 36 & 33 & 32 \\
\hline Rotación cartera Oficiales en días & 314 & 381 & 521 & 458 & 574 & 553 & 542 \\
\hline Eficiencia laboral $(\$ / \mathrm{m} 3)$ & $\frac{514}{182,57}$ & 217,38 & $\frac{21}{235,61}$ & 281,96 & 308,16 & 302,18 & $\frac{34 L}{315,66}$ \\
\hline Calldad del agua & $100 \%$ & $100 \%$ & $100 \%$ & $100 \%$ & $100 \%$ & $100 \%$ & $100 \%$ \\
\hline Continuidad del servicio & $94,4 \%$ & $93,4 \%$ & $95,2 \%$ & $98,2 \%$ & $99,8 \%$ & $99,9 \%$ & $100,0 \%$ \\
\hline \multicolumn{8}{|l|}{ Balance financiero } \\
\hline Ingresos (miles de US\$) ${ }^{*}$ & 6.701 & 7.783 & 9.032 & 10.385 & 12.188 & 14.134 & 7.298 \\
\hline Egresos (miles de US\$) & $\frac{0.71}{6.804}$ & 7.7066 & $\frac{9.032}{8.092}$ & $\frac{1.503}{10.021}$ & \begin{tabular}{|l|}
12.100 \\
11.178
\end{tabular} & 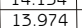 & 7.228 \\
\hline Utillidad o pérdida (miles de US\$)* & -103 & -182 & 940 & 363 & 1.010 & 160 & 70 \\
\hline
\end{tabular}

* Tipo de cambio equivalente: US\$ $1=\$ 2.340$ colombianos (Tasa representativa del mercado: Promedio Mayo 2005).

Fuente: Acuaviva - Departamento de Control y Desarrollo

Sin embargo, en este análisis no se incluyen los importantes incrementos tarifarios que han caracterizado el proceso de modernización del sector de A\&S en Colombia a partir de la expedición de la Ley de Servicios Públicos Domiciliarios en 1994 y que han afectado especialmente a los estratos más pobres de la población.

Por otra parte, al igual que en el esquema público, algunos de los servicios se siguen contratando externamente con consultores privados, tales como: reposición de obras de acueducto y alcantarillado; reparación y mantenimiento de vehículos de la empresa; arrendamiento de maquinaria y vehículos; recaudo de tarifas en bancos y supermercados y algunos pequeños diseños y estudios en general. Los servicios contratados externamente se pueden considerar como una política explícita de la empresa, basada en criterios de economía y búsqueda de servicios especializados (Acuaviva, 2001). Sin embargo, se resalta que la mayor parte del valor de las contrataciones corresponden a los contratos de asesorías ya comentados y que benefician a las firmas francesas constituidas para ese propósito, desplazando la contratación de mano de obra

\section{Volumen 7 - No. 1 - Julio de 2005}


calificada de las firmas regionales y locales que antes contrataba Empalmira. Igualmente, los contratos de artículos de ferretería que antes se hacían con los almacenes locales, ahora se hacen con grandes cadenas de la capital del Departamento (Cali), perjudicando al mercado local.

\section{CONCLUSIONES}

$\square$ La prestación de los servicios de A\&S en Colombia se ha caracterizado por mantener esquemas de asociación entre el sector público y el privado. En los inicios de los sistemas con conducción a la vivienda, la iniciativa estuvo a cargo del sector privado, para posteriormente ser asumidos por las autoridades locales dadas las limitaciones del primero para prestar los servicios con criterios de calidad y universalización. Sin embargo, los privados siempre han participado en el diseño y construcción de obras mediante consultoría externa. Esta situación se reproduce en la ciudad de Palmira.

ఐ En la coyuntura actual, aunque aún se considera pequeña la participación privada en Colombia en términos del número de organizaciones prestadoras (4,4\%), ésta es creciente y tiene mayor importancia en términos del número de población servida $(13,1 \%)$. Los esquemas que predominan son la Concesión y el Arrendamiento, resaltando una significativa participación de capital extranjero en medianas y grandes ciudades. adelantado en Palmira es complejo, lo cual dificulta el cumplimiento del papel de V\&C del socio propietario, el Municipio. Ello, junto a la desinstitucionalización del sector en el municipio producto de la liquidación de Empalmira e Infipal, tienen un efecto importante en la pérdida de gobernabilidad de la autoridad local sobre el manejo de sus servicios de A\&S.

$\square$ Los resultados de la negociación fueron altamente beneficiosos para las firmas francesas que participaron. La apropiación de recursos financieros que obtienen es elevada frente a los aportes realizados. Además, hay un balance negativo en términos de mejorar los flujos netos de ingresos foráneos que llegan al país.

घ En términos financieros, éste fue un mal negocio para el municipio. Aporta en capital mucho más de lo que obtiene. Además, sigue financiando parte de las inversiones con presupuesto del municipio, mostrando las limitaciones de los esquemas de Arrendamiento como instrumento para la reducción del déficit fiscal municipal.

$\square$ Los costos sociales para la localidad fueron importantes reflejados tanto en el incremento de tarifas como en el desplazamiento de cerca de 570 empleos, que fueron compensados con bonificaciones de liquidación y jubilaciones anticipadas, todas ellas financiadas con recursos de los ingresos de la negociación y el presupuesto municipal.

$\square$ Las observaciones sobre el proceso de contratación no pretenden desconocer la capacidad de las firmas extranjeras participantes, situación que se refleja en los altos niveles de gestión alcanzados, sino mostrar la necesidad de buscar procesos que brinden resultados más equilibrados para las partes. El objetivo de cualquier negocio debe ser la satisfacción de los participantes con relación a los beneficios recibidos, los cuales deben corresponder a sus aportes. Las relaciones Morte-Sur, deberán estar regidas por estos principios para que contribuyan a un mejor desarrollo, siendo el papel de los gobiernos y de las organizaciones internacionales, acompañar estos procesos de privatización para preservar los principios de equidad y justicia en bien del nivel local.

$\square$ Finalmente, cotejando esta experiencia con relación a lo planteado por las dos concepciones teóricas presentadas, encontramos lo siguiente: i) Existe evidencia que la prestación del servicio de A\&S 
realizado por Acuaviva presenta una mejora en los indicadores de gestión, con lo cual, se apoya uno de los argumentos planteados por la escuela defensora de los procesos de privatización en el sentido de que la misma mejorará la eficiencia y calidad en la prestación de los servicios. Sin embargo, esto no se puede generalizar para todos los casos como lo demuestran las experiencias de Cochabamba (Bolivia) y Buenos Aires (Argentina). ii) De la misma manera, buena parte de los argumentos sustentados por la visión crítica de la privatización también son corroborados. Los aportes reales de inversión del sector privado y del capital extranjero son bajos; la responsabilidad de las nuevas inversiones sigue recayendo en el presupuesto municipal, con lo cual el argumento de disminuir la presión fiscal estatal no es valido; la balanza de pagos de la inversión foránea resultó negativa, saliendo más dinero del país del que entró; ello es acompañado por el esquema de financiación del plan de inversiones el cual es soportado con recursos locales: presupuesto municipal, tarifas y crédito de la banca local; además, se produce un desplazamiento del conocimiento y recursos locales a favor de los recursos transnacionales afectando el desarrollo de la economía local y nacional. Igualmente, hay un balance negativo en la generación de empleo neto para el mercado laboral con el consecuente costo para las familias y los individuos expulsados de su trabajo. Por otra parte, aparecen visos de corrupción en el proceso de negociación realizado. Y finalmente, se produce un desmonte del aparato institucional local de $V \& C$ sobre la prestación de los servicios de A\&S, perjudicando en forma importante la gobernabilidad de las autoridades locales y el control de los usuarios. iii) Así, se puede concluir que en el balance general del proceso de participación del sector privado en la ciudad de Palmira, además de algunos beneficios en la mejora de los indicadores de gestión de la empresa, se producen también significativos costos sociales, económicos y financieros. Ello muestra la necesidad de hacer balances más neutrales de los procesos de privatización en el sector de A\&S para América Latina y Colombia, y no señalar sólo sus bondades sino resaltar también sus grandes costos y riesgos implícitos.

\section{BIBLIOGRAFÍA}

च Acuaviva (2001). Informes Planeación y Subgerencia Administrativa y Financiera. Palmira, Colombia.

■ Bakker, K. (2002). From state to market?: water mercantilization in Spain. En Environment and Planning. A, vol. 34, págs. 767-790.

च Betancourt, L.I. (1998). Avances y perspectivas de la regulación de los servicios públicos de agua potable y saneamiento básico. Mimeo, Bogotá.

घ Braadbaart, O., Blokland, M. y Schwartz, K. (1999). Taxonomy of modes of organization in the water sector. In: Public-Private partnerships and strategic managemet. UMESCO-IHE Institute for Water Education. Documento aún no publicado. Delft, The Metherlands.

च Comisión de Regulación de Agua Potable y saneamiento Básico, CRA (2001). El estado del arte de la regulación en el sector de agua potable y saneamiento básico en Colombia. Bogotá, Colombia.

च Cuervo, L. M. (1997). El agua potable como bien mayor. En Revista de la CRA, Regulación de Agua Potable y saneamiento Básico. Mo. 2, Bogotá, Colombia, Agosto.

च Dourojeanni, A. y Jouravlev, A. (1999). El Código de aguas de Chile: entre la ideología y la realidad. Serie Recursos Maturales e Infraestructura, CEPAL, Santiago de Chile.

ఐ Empalmira (1996). Informes varios de descripción del proceso de privatización. Palmira, Colombia.

\section{Volumen 7 - No. 1 - Julio de 2005}


च Empalmira (1992-1996). Informes financieros anuales. Palmira, Colombia.

च Fainboin I. y Rodríguez, C. (2001). El Desarrollo de la infraestructura en Colombia en la década de los noventa. Parte II. Serie Reformas económicas. CEPAL, Santiago de Chile.

$\square$ Franceys, R. (2000). Water and public-private partnershpis. En: Public-Private Partnerships and Strategic Management. UMESCO-IHE Institute for Water Education. Documento aún no publicado. Delft, The Metherlands.

च Goldman, M. (2003). Apuntes desde la cumbre de Johannesburgo: ¿haciendo historia?. En Ecología Política \# 24. Icaria, Barcelona, España.

ఐ Instituto UMESCO-IHE (1999). Institute for Water Education, Sector and Utility Management Group, 1999. En Public-Private Partnerships and Strategic Management, Delft, The Metherlands.

$\square$ Infipal (2001). Informe de gerencia. Palmira, Colombia.

ఐ Jaramillo, S. (1995). Ciento veinte años de servicios públicos domiciliarios en Colombia. Cinep, Bogotá, Colombia.

घ Lee, T. y Jouravlev, A. (2000). Private participation in the provision of water services. Serie Medio Ambiente y Desarrollo Mo. 2. CEPAL, Santiago de Chile.

$\square$ MIMDESARROLLO (2001). Balance sectorial. En: Sector de agua potable y saneamiento básico: retos y resultados, p.p. 48, Bogotá, Colombia.

■ Petras, J. y Veltmeyer, H. (2002). El imperialismo en el siglo XXI. La globalización desenmascarada. Edit. Popular, Madrid, España.

$\square$ Raffo, T. (1956). Palmira histórica. Imprenta Departamental, Cali.

$\square$ Raffo Rivera, A. (1992). De Llanogrande a Palmira. Edit. Cámara de Comercio Palmira.

ఐ República de Colombia (1994). Ley de Servicios Públicos Domiciliarios, 142 de 1994.

$\square$ www. banrepublica.gov.co

$\square$ www.dnp.gov.co 